\title{
Positionspapier Digitalisierung der Arbeitswelt
}

\author{
Oliver Sträter ${ }^{1} \cdot$ Klaus Bengler $^{2}$
}

๑) Springer-Verlag GmbH Deutschland, ein Teil von Springer Nature 2019

Die Globalisierung, die Digitalisierung und die Entwicklung hin zu einer Plattformökonomie verändern die Wertschöpfungsstrukturen in allen Wirtschaftszweigen. Diese Transformation geht mit einem grundlegenden Wandel der Arbeitswelt einher. Die daraus entstehenden Arbeits- und Organisationsformen bieten neue Chancen, flexibler, selbstbestimmter und kreativer zu arbeiten. Einerseits können mit Hilfe der Digitalisierung die Herausforderungen der demografischen Entwicklung auch über die Erwerbsarbeit hinaus gemeistert werden. Auf der anderen Seite können aber auch Entwicklungen ihren Lauf nehmen, die zu ungünstigen neuartigen Belastungssituationen im Arbeitsleben und geringerer betrieblicher Effizienz führen können.

Im Rahmen der Herbstkonferenz 2018 der GFA im Wissenschaftsjahr „Arbeitswelten der Zukunft“ wurden gemeinsam mit acatech wissenschaftliche Impulse für eine nachhaltige arbeitswissenschaftliche fundierte Arbeitsgestaltung mit Arbeitsforschenden und Führungskräften aus der Industrie diskutiert. Unter dem Titel „Der Mensch im Mittelpunkt - Innovationen für Arbeit mit Zukunft" beleuchtete die Herbstkonferenz das Thema Digitalisierung aus unterschiedlichen Perspektiven:

- Neue Herausforderungen: Arbeit in der digitalen Transformation

- Neue Perspektiven: Wandel der Arbeitsorganisation und Unternehmenskultur

- Neue Kollaboration: Innovationspotenziale der MenschMaschine-Interaktion

Prof. Dr. phil. habil. Oliver Sträter

straeter@uni-kassel.de

Prof. Dr. Klaus Bengler

bengler@tum.de

1 Fachbereich 15 - Institut für Arbeitswissenschaft und Prozessmanagement, Fachgebiet Arbeitsund Organisationspsychologie, Universität Kassel, Heinrich-Plett-Str. 40, 34132 Kassel, Deutschland

2 Lehrstuhl für Ergonomie, Technische Universität München, Boltzmannstr. 15, 85747 Garching b. München, Deutschland
- Neue Kompetenzen: Qualifizierung für die Bedarfe von morgen

Die Digitalisierung birgt große Potenziale, wirft gleichzeitig aber auch zahlreiche Fragen zu ihrer Gestaltung und Bewertung auf:

- Wie können und sollten zukünftige Arbeitswelten am Menschen orientiert gestaltet sein?

- Welche Gestaltungsfaktoren sind beim Einsatz von Zukunftstechnologien zu beachten, sodass sie im Sinn der Ergonomie effizient und sicher eingesetzt werden können?

- Wie können Eigenverantwortung und Selbstbestimmtheit des Einzelnen und Verantwortlichkeit von Unternehmen in ihrer Rolle als Arbeitgeber in eine gute Balance gebracht werden?

- Welche neuen Ansätze, Kooperationen und Strukturen sind im Bereich der Qualifizierung erforderlich?

- Wie können Wissenschaft und Forschung hierbei unterstützen?

Die Diskussionen auf der Konferenz zeigten die Wichtigkeit von arbeitswissenschaftlicher Expertise in der Gestaltung und Bewertung für eine nachhaltige, gesunde und effiziente Arbeitswelt der Zukunft auf.

Ganz wesentlich für eine menschengerechte digitale Transformation ist, dass die Arbeitsgestaltung nicht allein von der Technologie und der technischen Machbarkeit bestimmt werden darf, sondern die Arbeitsgestaltung im Sinn der Arbeitswissenschaft ausgehend von den menschlichen Bedarfen und Möglichkeiten durchzuführen ist. Eine solche, vom Menschen aus gedachte Arbeitsgestaltung ist eine Schlüsselvoraussetzung, damit die fortschreitende Digitalisierung zu sicheren, gesunden und effizienten Arbeitssystemen führt.

Sollte eine vom Menschen hergebrachte Arbeitsgestaltung nicht umgesetzt werden, wird das unweigerlich zu einer weiteren Verschärfung der ohnehin angespannten Belastungen der Menschen im Arbeitsleben führen. Das Thema der psychischen Belastung ist beispielsweise seit zehn Jahren immer stärker in Diskussion. Gerade die Zunahme in- 
formatorischer Tätigkeiten im Zug der Digitalisierung wirkt auf die Informationsverarbeitungsleistung des Menschen. Deshalb kann diese Entwicklung die Problematik hinsichtlich der psychischen Belastungssituation bei ungünstiger Gestaltung und Einbettung in der Organisation weiter verstärken.

Die Arbeit wird sich qualitativ verändern hinsichtlich der kognitiven Anforderungen an den Menschen und der Art der Zusammenarbeit. Die Veränderungen werden dazu führen, dass umfangreiche Anpassungen in der Qualifizierung und Qualifikationsstruktur erforderlich werden. Diese wiederum verlangen eine weitaus systematischere Planung und Koordination vor allem hinsichtlich der zeitlichen Abläufe von Technologieentwicklung, ergonomischen Anforderungen und Ausbildung. Ein systematischer Veränderungsprozess muss auf die Qualifikationssysteme wirken und strategisch/zeitlich den technischen Entwicklungen vorgelagert sein. Diese Veränderung muss vor allem die hohe Entwicklungsdynamik der Digitalisierungstechnologien aufgreifen. Die Digitalisierung wird wohl nicht zu einem Abbau von Arbeitsplätzen sorgen, aber für die zukünftige Wettbewerbsfähigkeit und die Realisierung ergonomischer Arbeitssituationen ausschlaggebend sein. Eine erhebliche Veränderung der erforderlichen Kompetenzen, die Umwandlung vieler Berufsbilder und das Entstehen neuer Berufsbilder werden die zentralen Anforderungen sein. Die Arbeitsforschung ist hier gefragt, diese Änderungsprozesse vor dem Hintergrund sehr dynamischer betrieblicher Veränderungen vorzubereiten.

Digitalisierung wird auch das Menschenbild verändern. Durch Mensch-Roboter-Kooperation und Mensch-RoboterKoexistenz besteht das Potenzial, Menschen durch Robotik nennenswert zu entlasten. Die Gestaltung dieser Arbeitssituationen muss aber zu Arbeitssituationen führen, die für die Beschäftigten kontrollierbar bleiben und in denen der Mensch „den Takt angibt“. Gerade für Systementwickler und Systemnutzer ist deshalb eine ethische Grundausbildung entscheidend, damit menschengerechte Arbeitsgestaltung auch gelingt. Ebenso besteht erheblicher Qualifizierungsbedarf bezüglich ergonomischer Inhalte für Entwickler robotischer Systeme, die mit Menschen kooperieren sollen. Der technische Gestaltungsprozess muss dabei insbesondere der Geschwindigkeit des Qualifizierungssystems und den Ausgangs-Fähigkeiten der Beschäftigten Rechnung tragen. Beschäftigten eine umfangreiche IT-Ausbildung zu geben, um ein digitales System adäquat nutzen zu können, benötigt Zeit und Aufbau einer entsprechenden Bereitschaft, diese IT-Systeme zu nutzen. Ein fundierter Prozess des psychologischen Änderungsmanagements ist dabei entscheidend für eine gute arbeitswissenschaftliche Gestaltung, aber auch für eine effiziente Nutzung digitalisierter Systeme. Prinzipien wie die Partizipation in Veränderungsprozessen oder rechtzeitige Einbindung einer entsprechen- den Qualifizierung sind erforderlich, damit die an digitale Systeme gerichteten Erwartungen passend und zeitgerecht erfüllt werden.

Damit haben im Rahmen der Digitalisierung arbeitswissenschaftliche Herangehensweisen auch einen sehr direkten Bezug zu betriebswirtschaftlichen Parametern. Durch eine mangelnde proaktive arbeitswissenschaftliche Gestaltung entsteht gerade in digitalen Systemen ein hohes Potenzial an Fehlinvestitionen durch unzureichend entwickelte Arbeitsmittel. Dies wird in einer sehr viel stärkeren Ausprägung stattfinden, als es in der klassischen Arbeitssystemgestaltung der Fall war. Denn das Potential für unergonomische Gestaltungslösungen nimmt allein durch die Komplexität digitaler Systeme und ihre Vernetzung stark zu. Bei sinnvoller arbeitswissenschaftlicher Gestaltung bieten diese Systeme jedoch auf der anderen Seite neue, auch betriebswirtschaftlich attraktive Lösungswege. Derzeit sind noch große Defizite in der grundlegenden softwareergonomischen Gestaltung vieler Systeme zu beobachten. Die stark zunehmende Interaktion des Menschen mit automatisierten und autonomen Systemen - seien es Algorithmen, Roboter oder automatisierte Fahrzeuge - finden derzeit noch häufig vor dem Hintergrund fehlender arbeitswissenschaftlicher Guidelines statt, die den Entwicklern wichtige Gestaltungshinweise geben würden.

Digitalisierung bedeutet dabei auch Veränderungen von Organisationsabläufen und -prozessen. Die klassischen wirtschaftlichen Ziele einer Organisation müssen mit der menschengerechten Arbeitsgestaltung vereinbart werden. Die Organisationsentwicklung muss eine proaktiv agierende Gestaltungskultur entwickeln und agile Führungsstrukturen zulassen.

Die Erwartungshaltung eines Betriebes durch Rationalisierungsstrategien im Rahmen der Digitalisierung produktiver zu werden steht der erhöhten psychischen Belastung gegenüber, die wiederum volkswirtschaftlich getragen bzw. kompensiert werden muss. Hier sind auch über das gesamte wirtschaftliche System sinnvolle Strategien zu entwickeln.

All diese Veränderungen im Nachhinein, durch eine reaktive korrektive Arbeitsgestaltung auszukurieren, wird nicht oder nur mit großem Aufwand möglich sein, obwohl bzw. gerade weil es sich um die Änderung komplexer digitaler Systeme handelt.

Die Diskussion im Rahmen der Konferenz zeigt, dass hier neue arbeitswissenschaftliche Methoden entstehen können und müssen. Es wird daher zu Parallelen in der pilotartigen Einführung neuer Arbeitsweisen und der Entwicklung des entsprechenden arbeitswissenschaftlichen Rahmens kommen. So wie Verfahren der künstlichen Intelligenz genutzt werden, um technologische Fortschritte zu erzielen, können ebensolche Verfahren auch genutzt werden, sehr viel intelligenter und gezielter arbeitswissenschaftliche Kenntnisse in die Gestaltung digitaler Systeme 
einfließen zu lassen. Ähnliches gilt für zukünftige Kompetenzbedarfe oder Qualifizierungsarten und Lernstrategien. Insgesamt ergeben sich für die Arbeitswissenschaft und die Arbeitsforschung folgende wichtige Herausforderungen, die zur Lösung der Probleme der Arbeitsgestaltung in der digitalen Transformation erforderlich sind:

- Eine proaktive Gestaltung von Arbeitssituationen in der Digitalisierung aus arbeitswissenschaftlicher Sicht ist einer reaktiven Gestaltung auf Basis technischer Machbarkeit vorzuziehen.

- Auf wissenschaftlicher Seite ist im Sinn eines neuen Referenzsystems die Entwicklung eines ökologisch validen Ansatzes zur Analyse und Bewertung der physischen und psychischen Auswirkungen von Digitalisierung erforderlich, der in der Lage ist, die komplexen Effekte der Digitalisierung zu beschreiben. Nur hierdurch wird eine proaktive Arbeitsgestaltung ermöglicht.

- Die proaktive Gestaltung erfordert entsprechend frühzeitig ansetzende Planungsverfahren bzw. die Nutzung der vorhandenen arbeitswissenschaftlichen Instrumente in den frühen Phasen der Arbeitsgestaltung; diese Planungsverfahren sind nicht allein technischer Natur, sondern beziehen arbeitswissenschaftliche Erkenntnisse, ethische Grundsätze und gesellschaftliche Anforderungen mit ein.

- Konzipierung und Implementierung einer sowohl zeitlich als auch inhaltlich auf die Technologieentwicklung abgestimmten Qualifizierung der Personen, die digitale Systeme nutzen und zusätzlich auch derjenigen, die digitalisierte Systeme erstellen. Hierbei bestehen inhaltliche Anforderungen hinsichtlich der arbeitswissenschaftlichen und ethischen Erkenntnisse; zeitliche Anforderungen bestehen darin, dass rechtzeitig und proaktiv der nötige Qualifizierungsumfang und die nötige Qualifizierungsdauer (d.h. beispielsweise bereits in der universitären Ausbildung) berücksichtigt werden.

- Die sehr dynamische technische und gesellschaftliche Entwicklung erfordert einen kontinuierlichen Dialogprozess mit Fokus auf die Arbeitsforschung.

- Die Umsetzung des Transformationsprozesses mit zentraler Einbindung arbeitswissenschaftlicher Expertise soll auch dazu dienen, in Pilotanwendungen Best Practice Beispiele zu erzeugen, die dazu dienen können, allgemeine Ansätze zu formulieren. 